\title{
Perceptual grouping produced by line figures
}

JACOB BECK

UNIVERSITY OF OREGON

Two experiments, one with 2 and one with 3-line figures, studied the relative effectiveness of differences in orientation and shape in producing grouping by similarity. The results showed that changes in shape or orientation which leave the component lines of the figures vertical and horizontal do not facilitate grouping as readily as changes which alter the direction of the component lines to $45^{\circ}$ and $135^{\circ}$. These results corroborate and extend the findings of Beck (1966a, 1966b) and are discussed in relation to the problem of specifying the properties of line figures that produce grouping by similarity.

In an earlier study Beck (1966a) used a method based upon threshold measurements to test the effectiveness of changes in orientation and shape of line figures in producing grouping by similarity. Two sets of figures, for example, a pattern of upright Ts (A in Fig. 1) and a pattern of Ts tilted at $45^{\circ}$ (B in Fig. 1), were combined so that a single equally spaced field was given. When the brightness of the two patterns was equal, there was no separation of the field into two groups of upright and tilted Ts. However, when the brightness of the pattern of upright $T$ s was reduced, the perceptual field separated into two groups-the less bright upright Ts and the brighter tilted Ts. Measurements of the amount by which the luminance of the upright $T s$ had to be reduced in order for the figures to segregate into two perceptual groups permitted an evaluation of the relative effectiveness of changes in shape and orientation in producing perceptual grouping. The results showed that changes in shape or orientation which leave the component lines of the figures vertical and horizontal do not facilitate grouping as readily as changes which alter the direction of the component lines to $45^{\circ}$ and $135^{\circ}$.

When the brightness of the upright Ts was reduced, separation of the field into two groups, however, did not occur suddenly but gradually. Since the experiments required that os maintain a stable criterion for the point at which the field was judged to separate into two groups, six highly practiced Os served in the experiments. The present study examines the generality of the earlier results. Two separate groups of naive Os served under changed experimental procedures. Instead of asking $O s$ to reduce the brightness of the upright $T s$ to the point where perceptual grouping occurred, the Os were now shown the combined field with the upright $T$ s set at three lower luminance levels. The os task was to scale the clarity with which the combined field separated into two groups when the upright Ts were set at each of these three luminance levels. Two independent experiments, one with 2 and one with 3-line figures, examined the relative effectiveness of changes in shape and orientation in producing perceptual grouping.

\section{Apparatus}

\section{METHOD}

The stimulus display in Experiments 1 and 2 consisted of an equally spaced field, $9.9 \times 10.4$ in., projected onto a smooth black wall 37 in. from the $O$. Each field was composed of two patterns of figures, a standard pattern and a comparison pattern. Two matched projectors were used, a slide of the standard pattern was placed in one projector and slides of the comparison patterns were placed in the other. The standard pattern, which was always present, consisted of 40 upright Ts and is shown as $A$ in Fig. 1. The comparison patterns consisted of 24 figures and were varied. $B$ in Fig. 1 shows a pattern of Ts tilted at $45^{\circ}$, one of a larger number of comparison patterns used in this study. When the patterns were of equal brightness, an $O$ saw a single field made up of two kinds of luminous figures against a black background. When the standard pattern was set at luminances lower than that of the comparison pattern, the field tended to segregate into two groups of figures. The brightness of the standard pattern was set at three lower levels by means of a Variac. A red Wratten filter, number 29, was placed in each projector to minimize color temperature changes.

Luminance measures were taken with a SpectraPritchard photometer, employing a 30-min arc field. The meter was placed at 90 in. from the targets and subtended a circular field of approximately $.8 \mathrm{in.} \mathrm{in}$

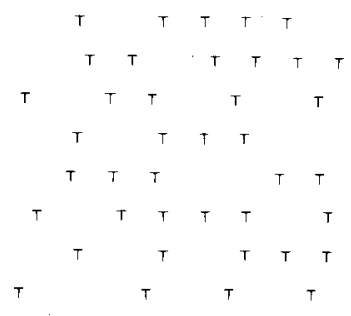

A

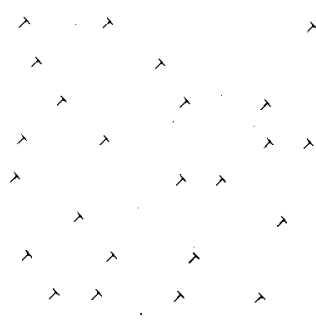

B
Fig. 1. Sample displays used in experiments. (A illustrates the pattern of upright $T$ s employed as the standard in Experiments 1 and 2. B illustrates a pattem of Ts tilted at $45^{\circ}$, one of a larger number of comparison pattems used. The two pattern's were combined so that a single equally spaced field of upright and tilted Ts. was obtained. In the experiments, the patterns consisted of red figures against a black background.) 
diameter. The luminance of each figure averaged over this aperture was measured. All luminance measures refer to the mean luminance of the figures making up a pattern.

\section{Stimuli for Experiment 1}

In Experiment 1, nine comparison patterns composed of 2-line figures were used. The lines of each figure were .3 in. long and .031 in. wide; they were always at right angles to each other. The figures making up each pattern were first made by affixing a selfsticking black tape to matte white cardboard. Reversal film was used to prepare slides in which the white background became opaque and the black lines became transparent. In the photographic process great care was taken to insure that the lines composing the different figures would be equal in brightness. The nine comparison patterns were: (a) an upright $T$, (b) a $T$ on its side, (c) an inverted $T,(d)$ an upright $T$ rotated $45^{\circ}$, (f) a + , (g) a + rotated $45^{\circ}$ to form an $X$, (h) a backward $L$, and (i) the $L$ shape rotated $45^{\circ}$ to form a V. (The exact shapes are given in Fig. 2.) The two lines making up each figure, when projected, were .3 in. and the separation between adjacent figures when the standard and comparison patterns were combined was approximately $.85 \mathrm{in}$. The luminance of the comparison patterns was always set at $.07 \mathrm{ft}-\mathrm{L}$. The luminance of the standard pattern was set at .039 , .032 , and $.025 \mathrm{ft}-\mathrm{L}$. The nine comparison patterns, when combined with the standard pattern and presented at each of these three luminance levels, made a total of 27 stimulus displays. The 27 stimulus displays were presented to each $O$ in a different random order, with the constraint that each of the patterns appear once in every block of nine trials.

\section{Stimuli for Experiment 2}

In Experiment 2, four comparison patterns composed of 3-line figures were used. All lines were again .3 in. in length and at right angles to one another. The patterns were prepared and projected as in Experiment 1. They were: (a) an upright $F,(b)$ an $F$ on its side, (c) an $F$ rotated $45^{\circ}$ and (d) an upright $H$. (See Fig. 3.) The brightness of the individual lines composing the figures was visually matched to the brightness of the lines in Experiment 1. Since this experiment used 3-line instead of 2-line figures, the average luminance within the aperture was now .1 ft-L. The standard pattern again consisted of upright $\mathrm{Ts}$, this time set at $.057, .047$, and $.039 \mathrm{ft}-\mathrm{L}$. The four comparison patterns, when combined with the standard and presented at each of these three luminance levels, made a total of 12 stimulus displays. The 12 stimulus displays were again presented in a different random order to each $O$, subject to the constraint that each of the patterns appear once in every block of four trials.

\section{Procedure}

There was no light in the room in which the experiments were conducted, other than that coming from the projectors. The Os viewed the stimuli binocularly. No chin rest was employed. The general procedure is best described by the instructions given. The instructions (slightly abridged to eliminate some of the examples given) in Experiment 1 were:

I will show you a series of displays all composed of red figures. Each of the displays you will see is composed of two separate patterns; this one (the $O$ was shown the pattern of upright Ts) will be present in all displays; this one (the $O$ was shown the pattern of Ts on their side) will change from trial to trial. I am interested in finding out how much these patterns stand out relative to each other when they are presented together (the $O$ was shown the standard pattern of upright Ts together with the comparison pattern of Ts on their side). As the shapes are different, it is easy to pick out the figures that are different. This, however, is not the judgment I wish you to make. Rather, I would like you to indicate how clearly one of the patterns as a whole groups stands out relative to the other. An exaggerated example of what I mean is this. (The pattern of upright Ts was set at $.015 \mathrm{ft}-\mathrm{L}$; the pattern of Ts on their side at $.07 \mathrm{ft}-\mathrm{L}$.$) In the experiment, you will never$ see this extreme an example, but as you see, all the $T s$ on their side stand out as a complete pattern, separate from the upright $T s$. In this case this does not happen. (The $O$ was shown the pattern of upright Ts and Ts on their side at .07 ft-L.) I would like you to indicate the degree to which the two patterns are seen as separate groups, by means of a 6-point rating scale, where 0 indicates that the two patterns cannot be seen as separate, but are seen as one display with differences in shape; and 5 indicates that they are very clearly seen as two separate groups. During the experiment a given pattern will be repeated a number of times, but no pattern will be shown more than once under the same conditions. This means there will be no inconsistency involved in assigning a value of 1 to a pattern in one trial and later assigning a value of 5 , for example, to the same pattern in another trial.

In order to familiarize Os with the stimuli, the entire set was shown before the experiment began.

The instructions in Experiment 2 were the same as in Experiment 1 except that the examples presented were now chosen from the comparison patterns used in Experiment 2.

\section{Ohservers}

The Os were summer school students at Harvard University who were paid to participate. Two separate 


\section{EXPERIMENT 1}

\begin{tabular}{|c|c|c|c|c|c|c|c|}
\hline & & AN & RATIN & S & $=.039$ & ft. & \\
\hline $\begin{array}{l}T \\
0.42\end{array}$ & $\begin{array}{l}T+ \\
1.05\end{array}$ & $\begin{array}{l}T \vdash \\
1.26\end{array}$ & $\begin{array}{l}T \times \\
1.42\end{array}$ & $\begin{array}{l}T \perp 1 \\
1.47\end{array}$ & $\begin{array}{l}T\lrcorner \\
1.53\end{array}$ & $\begin{array}{l}T Y \\
247\end{array}$ & $\begin{array}{l}T V \\
2.95\end{array}$ \\
\hline
\end{tabular}

\begin{tabular}{lllllllll}
\multicolumn{10}{c}{ MEAN RATINGS } & $=.032$ & $\mathrm{ft}-\mathrm{L}$. \\
$\mathrm{TT}$ & $T+$ & $T \vdash$ & $T \times$ & $T \perp$ & $T \perp$ & $T V$ & $T Y$ & $T \lambda$ \\
105 & 1.68 & 179 & 1.84 & 2.05 & 2.63 & 2.95 & 3.32 & 3.58
\end{tabular}

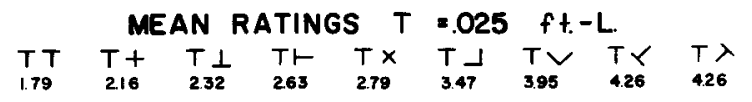

MEAN RATINGS DISREGARDING LUMINANCE

$T T T+T r T \perp T \times T \perp T \vee T Y T \lambda$

$\begin{array}{llllllllll}3.26 & 4.89 & 5.68 & 5.84 & 6.05 & 783 & 0.87 & 10.05 & 10.99\end{array}$

Fig. 2. The mean ratings of the degree to which the standard and comparison pattems were perceived to group in Experiment 1. (The first figure in each pair of figures shown illustrates the shape of the figures composing the standard pattem; the second one, the shape of the figures composing the comparison pattern. The luminance of the standard pattem is indicated above each row. The rated grouping disregarding luminance and averaged over $O$ s is shown in the bottom row. A Tukey test of multiple comparisons was used to compare the means. Those means which do not differ significantly from each other at the .01 level are underscored by the same line.)

groups of Os made judgments. Nineteen Os served in Experiment 1 and 14 Os served in Experiment 2.

\section{RESULTS}

Figures 2 and 3 summarize the data obtained in Experiments 1 and 2. In these figures, the luminance of the standard pattern is indicated above each row. The first flgure in each pair of figures shown illustrates the figures composing the standard pattern and the second one the figures composing the comparison pattern. The number below each pair of figures is the mean rating of the degree to which the standard and comparison patterns were perceived to group. The comparison patterns have been arranged so that they go from the least to the greatest amount of grouping. The grouping produced by the comparison patterns, disregarding the luminance of the standard, can be obtained by totaling the mean ratings at each of the three luminance levels. They are shown in the bottom rows in Figs. 2 and 3. These numbers represent the scores obtained when the ratings given a comparison pattern at each luminance level are summed for each $O$ and the total of the sums divided by the number of Os.

The overall effects of Figure Shape and Luminance in producing grouping are obtained from a 3 by 3 analysis of variance. Tables 1 and 2 show that the factors of Figure Shape and Luminance are significant at beyond the .001 level in both Experiments 1 and 2 . The Figure by Luminance interaction is significant at the .005 level in Experiment 1 and the .05 level in Experiment 2. Even though the interaction was significant, examination of Figs. 2 and 3 show that the treatment factors have the same rank or approximately the same rank for each level. As the luminance of the standard pattern is decreased, rated grouping increases for each comparison pattern. At each luminance level, the rank order of the grouping produced by the different figures is approximately the same. This homogeneity reflects the fact seen in Tables 1 and 2 that the mean squares for Luminance and Figure Shape are very much larger than the mean squares for their interaction (Lindquist, 1953, p. 143).

In order to compare the degree to which different figures produce grouping, a Tukey test of multiple comparisons was applied to the overall mean ratings. The means that do not differ significantly from each other at the .01 level are underscored by the same line in Figs. 2 and 3. The results show that in both Experiments 1 and 2 changes in the orientation or shape of the figures without changes in the orientation of the component lines do not as readily facilitate the separation of the field into distinct perceptual groups. In Experiment 1, the comparison patterns, $T$ on its side and inverted $T$, involved differences in the orientation of the figures and the comparison pattern backward $L$ involved a difference in the shape of the figures, but in both cases the component lines remained vertical and horizontal; the comparison patterns $T$ rotated $45^{\circ}$, inverted $T$ rotated $45^{\circ}$, and $V$ involved changes in orientation and shape in which the component lines are tilted rather than vertical and horizontal. Figure 2 shows that the tilted figures differ

\section{EXPERIMENT 2}

$$
\begin{aligned}
& \text { MEAN RATINGS } T=.057 \mathrm{ft}-\mathrm{L} \\
& \begin{array}{llll}
T T & T F & T H & T \widehat{T} \\
1.71 & 1.86 & 207 & 380
\end{array}
\end{aligned}
$$

$$
\begin{gathered}
\text { MEAN RATINGS T=.047 } \mathrm{ft}-\mathrm{L} \\
T T T H \text { TF T^ }
\end{gathered}
$$$$
\begin{gathered}
\text { MEAN RATINGS } T=039 \mathrm{ft}-\mathrm{L} \\
\text { TH THT TF T^ }
\end{gathered}
$$$$
\begin{array}{llll}
T H & T T & T F & T \hat{~} \\
2.92 & 3.14 & 329 & 4.43
\end{array}
$$

\section{MEAN RATINGS DISREGARDING LUMINANCE \begin{tabular}{cccc}
$T \prod$ & $T H$ & $T F$ & $T \widehat{A}$ \\
678 & 735 & 772 & 12.07 \\
\hline
\end{tabular}}

Fig. 3. The mean ratings of the degree to which the standard and comparison pattems were perceived to group in Experiment 2 . (The first figure in each pair of figures shown illustrates the shape of the figures composing the standard pattem; the second one, the shape of the figures composing the comparison pattem. The luminance of the standard pattem is indicated above each row. The rated grouping disregarding luminance and averaged over $O s$ is shown in the bottom row. A Tukey test of multiple comparisons was used to compare the means. Those means which do not differ significantly from each other at the .01 level are underscored by the same line.) 
Table 1. Summary of the Analysis of Variance of Os Judgments of Grouping in Experiment 1

\begin{tabular}{lcccc}
\multicolumn{2}{l}{ Source of Variation } & df & MS & $F$ \\
\hline Figures & (F) & 8 & 43.7 & $23.00 * * *$ \\
Luminances & $(\mathrm{L})$ & 2 & 76.4 & $58.77^{* * *}$ \\
Observers & $(\mathrm{O})$ & 18 & 18.6 & ---- \\
F X & 16 & .87 & $2.81^{* *}$ \\
$F \times O$ & 144 & 1.9 & ---- \\
LXO & 36 & 1.3 & --- \\
$F \times L \times 0$ & & 288 & .31 & ---- \\
Total & 512 & & \\
\hline
\end{tabular}

$* * * p<.001$

$* * \quad p<.005$

significantly at the .01 level from the vertical-horizontal figures but not between themselves. In Experiment 2, Fig. 3 shows that an $F$ tilted at $45^{\circ}$ grouped significantly better than either an upright $F$, an $F$ on its side, or an upright $\mathrm{H}$.

As in the earlier study of Beck (1966a), not all $45^{\circ}$ rotations facilitated grouping. A + rotated $45^{\circ}$ to form an $X$ has the same tilt as the lines of a tilted $T$ and $V$, yet it groups less readily. It should also be noted that not all vertical and horizontal arrangements of lines produce the same grouping. The backward L grouped better than the other figures with vertical and horizontal lines at each of the three luminance levels. The Tukey test shows that it differed significantly from both the upright figures and from the rotated figures. The results also indicate that the addition of a line to both the upright and the tilted figures facilitates perceptual grouping. When the luminance of the standard pattern was set at $.039 \mathrm{ft}-\mathrm{L}$, the rated groupings of the comparison patterns are consistently higher in Experiment 2 than in Experiment $1(p=.021$, Fisher exact probability test).

\section{DISCUSSION}

Since Wertheimer (1923), psychologists have pointed out that similarity serves as a basis for perceptual grouping. An examination of similarity relationships, however, reveals that not all kinds of similarity produce grouping equally well. The present results corroborate the findings of Beck $(1966 \mathrm{a}, \mathrm{b})$ that line orientation is an important variable. If the orientation of a figure's lines are changed to $45^{\circ}$ and $135^{\circ}$, perceptual grouping relative to figures with vertical and horizontal lines will be improved. Changes in figure orientation or shape, however, which maintain a vertical and horizontal orientation of the component lines do not as readily facilitate grouping. Beck $(1966 \mathrm{~b})$ has shown that the decisive variable is the tilt of the lines and not changes in the vertical and horizontal dimensions which accompany changes in figure orientation. Beck (1966a, b) also showed that the rated similarity of the figures is not a good predictor of the degree to which figures will cohere to form perceptual groups.
Table 2. Summary of the Analy sis of Variance of Os Judgments of Grouping in Experiment 2

\begin{tabular}{|c|c|c|c|c|}
\hline \multicolumn{2}{|c|}{ Source of Variation } & \multirow{2}{*}{$\frac{d f}{3}$} & \multirow{2}{*}{$\frac{M S}{27.4}$} & \multirow{2}{*}{$\frac{F}{28.25^{* * *}}$} \\
\hline Figures & $(F)$ & & & \\
\hline Luminances & (L) & 2 & 19.1 & $32.93 * * *$ \\
\hline Observers & (0) & 13 & 4.23 & --- \\
\hline$F \times L$ & & 6 & .74 & $2.31 *$ \\
\hline$F \times O$ & & 39 & .97 & ---- \\
\hline $\mathrm{L} \times \mathrm{O}$ & & 26 & .58 & $-\ldots-$ \\
\hline$F \times L \times O$ & & 78 & .32 & ---- \\
\hline Total & & 167 & & \\
\hline
\end{tabular}

$* * * p<.001$

$* \quad p<.05$

Though going beyond the scope of the data, a brief discussion of two questions pertaining to the results is useful. Though an individual is sensitive to many pattern differences, not all differences that an individual can discriminate seem capable of producing perceptual grouping. The two pairs of figures $\mathrm{T}$ and $\mathrm{T}$ on its side and $T$ and $T$ tilted $45^{\circ}$ viewed individually are equally discriminable. Yet, when one is presented with a large number of figures the field $T$ and $T$ tilted $45^{\circ}$ groups readily while the field $T$ and $T$ on its side fails to group (1966b). A characterization of the variables that facilitate grouping is important. If one assumes that different properties of figures are responded to at different neural levels of the visual system, it is then possible that the processes involved in grouping are most sensitive to those properties that are selectively responded to at an early stage in the visual system. What is suggested is that the processes in grouping are based on a spontaneous direct response to relatively simple properties such as brightness, size, and line direction. Perhaps the reason that differences in figural orientation and figural similarity do not produce strong grouping is that these are observed through the mediation of a higher less spontaneous inspection process. The greater grouping produced by a backward $\mathrm{L}$ in Experiment 1 suggests that the presence of a right angle facilitates grouping. This property, however, did not appear in an earlier study (1966a). The basic importance of line orientation is consistent with Gibson (1950) who has suggested that the direction of a line is a basic element in the perception of a figure and Hubel and Wiesel (1962) who found specific receptors for line orientation when studying the cortical neurons in Area 17. This interpretation of the results is of course speculative and provisional until verified by further experiments.

A second question concerns why the figure $X$, in which the component lines of a + have been tilted at $45^{\circ}$ and $135^{\circ}$ does not group as well as a tilted $T$ or $V$. One explanation is that the visual system is sensitive to the orientation of the overall distribution of brightness of the total figure. If we assume that the visual system "averages" brightness over the whole 
area of a figure (such as the distribution of brightness obtained when the figure is out of focus) the orientation of this brightness distribution as well as the orientation of the component lines of a figure may be important. An upright $T$ and a tilted $T$ differ not only in the direction of their lines but also in the direction of the distribution of brightness. The distributions of brightness for $a+$ and an $X$ because of their symmetry are much more similar to each other and to an upright $T$. It should be pointed out that the improved grouping produced by the addition of a line in Experiment 2 may be the result of an increased difference in the overall brightness of a figure as well as due to a difference in the number of lines composing a figure. Independent variation of the average brightness of a figure and the number and arrangement of lines in a figure is especially difficult and caution is, therefore, required in assessing the relative importance of these variables.

\section{References}

Beck, J. Perceptual grouping produced by changes in orientation and shape. Science, 1966a, 154, 538-540.

Beck, J. Effect of orientation and of shape similarity on perceptual grouping. Percept. \& Psychophys., 1966b, 1, 300-302.

Gibson, J. J. The perception of the visual world. Cambridge: Riverside Press, 1950.

Hubel, D. H., \& Wiesel, T, N. Receptive fields, binocular interaction, and functional architecture in the cat's visual cortex. J. Physiol, 1962, 160, 106-123.

Lindquist, E. F. Desion and analysis of experiments in psychology and education. Cambridge: Riverside Press, 1953.

Wertheimer, M. Untersuchungen zur Lehre von der Gestalt, II. Psychol. Forsch, 1923, 4, 301-350.

\section{Note}

1. This research was supported by National Science Foundation Grant GB-2901. The preparation of this paper was supported by National Science Foundation Grant GB-5285.

(Accepted for publication June 27, 1967.) 